\title{
MIMO Ricean Channel Capacity
}

\author{
Guillaume Lebrun, Michael Faulkner \\ Australian Telecommunication CRC \\ Centre for Telecom. and Micro-electronics \\ Victoria University, Melbourne, Australia \\ email: \{lebrun,mf $\} @$ sci.vu.edu.au
}

\author{
Mansoor Shafi \\ Telecom New Zealand Ltd \\ 49-55 Tory Street \\ Wellington, New Zealand \\ email: mansoor.shafi@telecom.co.nz
}

\author{
Peter J. Smith \\ Dept. of Electrical and Computer Eng. \\ University of Canterbury \\ Private Bag 4800 \\ Christchurch, New Zealand
}

\begin{abstract}
This paper presents asymptotic bounds and limits for the ergodic channel capacity of MIMO systems under Ricean channel conditions. It is shown that the ergodic capacity per dimension decreases as the $K$ factor increases in value and approaches a value equal to that of the underlying scattering channel when the number of antennas are large. The accuracy of the bounds is verified by simulations. In addition, a variety of results for the MIMO Ricean channel are brought together to give an overview of the current knowledge in this area.
\end{abstract}

\section{INTRODUCTION}

Since the work of Foschini [1] and Telatar [2], there has been intense research activity in the area of MIMO systems. Most of this research effort has been focussed on the flatfading Rayleigh channel, which corresponds to a wireless propagation environment where the number of scatterers is large. It is now well understood that the capacity of the MIMO Rayleigh channel increases linearly with the number of antennas for a fixed ratio of transmit to receive antenna numbers. Therefore, it is convenient to define a normalized capacity, the capacity of the channel divided by the minimum of the number of antennas at the receiver and the number of antennas at the transmitter.

It has been reported recently that the standardized Rayleigh channel capacity tends to a Gaussian random variable as the number of transmit and receive antennas tends to infinity [3]. Further, the capacity distribution is close to Gaussian even for small antenna numbers. A good summary of asymptotic results for the independent Rayleigh case is given in [4].

In this paper we consider the ergodic capacity for the more general case of a Ricean channel. In the Ricean case, the flat-fading channel is composed of a Line Of Sight (LOS) component and a Rayleigh component. The choice of the Ricean $K$-factor varies the Ricean channel from a Rayleigh channel $(K=-\infty \mathrm{dB})$ to a pure LOS channel $(K=+\infty$ $\mathrm{dB})$. It has been shown [5] that the MIMO capacity decreases with increasing power of the LOS component (the $K$-factor). Related work is found in [6] and [7].

The outline of the paper follows: the system model is described in Section II. The capacity of LOS and Rayleigh channels is discussed in Section III. Section IV presents the main contribution of this paper: we show that the ergodic normalized capacity of the Ricean MIMO channel approaches the corresponding normalized capacity of the underlying scattering channel when the antenna numbers are large. Simulation results are presented to confirm the accuracy of this result.

\section{SYSTEM MODEL}

Consider a single-user MIMO system. Transmission is over a flat-fading Ricean channel with $t$ antennas at the transmitter and $r$ antennas at the receiver. If $\boldsymbol{x}$ is a vector of the input symbols $\left(\boldsymbol{x} \in \mathbb{C}^{t}\right), \boldsymbol{H}$ the channel matrix $\left(\boldsymbol{H} \in \mathbb{C}^{r \times t}\right)$, and $\boldsymbol{n}$ a vector of additive white Gaussian noise (AWGN) on the receiving antennas $\left(\boldsymbol{n} \in \mathbb{C}^{r}\right)$, the vector of received symbols can be expressed as

$$
\boldsymbol{y}=\boldsymbol{H} \boldsymbol{x}+\boldsymbol{n} .
$$

In Ricean fading the elements of $\boldsymbol{H}$ are non-zero mean complex Gaussians. Hence we can express $\boldsymbol{H}$ in matrix notation as [6]

$$
\boldsymbol{H}=a \boldsymbol{H}^{s p}+b \boldsymbol{H}^{s c}
$$

where the specular and scattered components of $\boldsymbol{H}$ are denoted by superscripts, $a>0, b>0$ and $a^{2}+b^{2}=1$. The entries of $\boldsymbol{H}^{s c}=\left(h_{i, j}\right)$ are independent and identically distributed (iid) complex Gaussian random variables with zero mean and unit magnitude variance. A common model for $\boldsymbol{H}^{s p}$ is $\boldsymbol{H}^{s p}=$ $\boldsymbol{v}_{r} \times \boldsymbol{v}_{t}^{*}$ [6] where $\boldsymbol{v}_{r}$ and $\boldsymbol{v}_{t}$ have modulus one elements and are the specular array responses at the receiver and the transmitter respectively. Here, we assume $\boldsymbol{H}^{s p}$ is an $r \times t$ matrix of unit entries since this definition gives exactly the same distribution for the eigenvalues of $\boldsymbol{H} \boldsymbol{H}^{*}$ as the standard model. The superscript * denotes the transpose conjugate. The Ricean $K$-factor is defined as $10 \log _{10}\left(a^{2} / b^{2}\right) \mathrm{dB}$.

Assuming equal power uncorrelated sources the capacity is

$$
C=\log _{2}\left(\left|I_{r}+\frac{\rho}{t} \boldsymbol{H} \boldsymbol{H}^{*}\right|\right),
$$

where $I_{r}$ is the identity matrix of dimension $r, \rho$ is the SNR on each receiving antenna and $|$.$| denotes the determinant$ of a matrix. The ergodic capacity is commonly defined as the expected value of $C$ in (3). The normalized capacity is defined as the ergodic capacity divided by $m$ where $m \triangleq$ $\min (t, r)$. Although this paper considers the baseline case of equal power transmission, more complex systems might use channel information to vary these powers according to some performance criterion. 


\section{CAPACITY OF RAYLEIGH AND LOS ChanNElS}

In this Section we summarize results on the extreme cases of a pure LOS channel and a pure Rayleigh channel. Some new results are also given to complete the summary.

\section{A. Pure LOS Channel : $K=+\infty$}

In general, a MIMO LOS channel has a capacity of

$$
C(K=+\infty, t, r, \rho)=\log _{2}(1+\rho r) .
$$

Since the channel is not random, the capacity is fixed and the ergodic capacity and the capacity are equal. It should be noted that the capacity does not depend on the number of transmit antennas, and only increases logarithmically with the number of receive antennas. In the special case $t=r=1$, the channel reduces to a Single Input Single Output (SISO) Additive White Gaussian Noise (AWGN) channel.

\section{B. Pure Rayleigh Channel : $K=-\infty, r=1$ or $t=1$}

For the Rayleigh channel, $\left\|\boldsymbol{H}_{i, j}\right\|^{2}$ is a $\chi_{2}^{2}$ variate (chisquared variate with two degrees of freedom) but normalized so that $E\left(\left\|\boldsymbol{H}_{i, j}\right\|^{2}\right)=1$, where $E($.$) denotes the expectation$ and $\|$.$\| denotes the absolute value. For one transmit antenna,$ the channel capacity is [1]

$$
C(K=-\infty, t=1, r, \rho)=\log _{2}\left(1+\rho \chi_{2 r}^{2}\right),
$$

and using one receive antenna the channel capacity is [1]

$$
C(K=-\infty, t, r=1, \rho)=\log _{2}\left(1+(\rho / t) \chi_{2 t}^{2}\right) .
$$

Notice that

$$
\begin{aligned}
E\left(1+(\rho / t) \chi_{2 t}^{2}\right) & =(1+\rho) \\
E\left(1+\rho \chi_{2 r}^{2}\right) & =(1+\rho r),
\end{aligned}
$$

and $\log _{2}(\cdot)$ is a convex function, that is $\forall z>0 E\left(\log _{2}(z)\right) \leq$ $\log _{2}(E(z))$. Therefore

$$
E(C(K=-\infty, t, r=1, \rho)) \leq E(C(K=+\infty, t, r=1, \rho))
$$

and

$E(C(K=-\infty, t=1, r, \rho)) \leq E(C(K=+\infty, t=1, r, \rho))$,

Hence, for a Single Input Multiple Output (SIMO) or Multiple Input Single Output (MISO) channel, the ergodic capacity is higher in a LOS case than in a Rayleigh case (see Fig. 1).

C. $K=-\infty, r \rightarrow \infty, t \rightarrow \infty$ and $t / r=\alpha$

When $\boldsymbol{H}$ is Rayleigh $(K=-\infty)$ and the number of antennas is large, the normalized capacity can be approximated by a Gaussian random variable [3]. Suppose $r \rightarrow \infty, t \rightarrow \infty$ with $t / r=\alpha$, then the mean is given by [4]

$$
\begin{aligned}
E(C / m)= & \left(\log _{2}\left(w_{+} \rho\right)+\ldots\right. \\
& \left.\left.(1-\alpha) \log _{2}\left(1-w_{-}\right)-\frac{w_{-} \alpha}{\ln 2}\right) \max (1, \beta)\right) .
\end{aligned}
$$

where $m \triangleq \min (t, r), \beta \triangleq 1 / \alpha$,

$$
w_{ \pm} \triangleq\left(w \pm \sqrt{w^{2}-4 / \alpha}\right) / 2
$$

and

$$
w \triangleq 1+\frac{1}{\alpha}+\frac{1}{\rho} .
$$

The variance of $\mathrm{C}$ is also given in [4] as,

$$
\sigma_{C}^{2}=-\log _{2} e \log _{2}\left\|1-\frac{q^{2} p^{2}}{\beta}\right\|
$$

with

$$
\begin{aligned}
& q \triangleq \frac{\sqrt{\rho}}{2}\left(\beta-1-1 / \rho+\sqrt{(\beta-1-\rho)^{2}}+4 \beta / \rho\right) \\
& p \triangleq \frac{\sqrt{\rho}}{2}\left(1-\beta-1 / \rho+\sqrt{(1-\beta-\rho)^{2}}+4 / \rho\right) .
\end{aligned}
$$

From these results, it is obvious that the mean capacity grows linearly with the number of antennas.

\section{Asymptotic CAPACity of A RicEAN CHANNEL}

While the capacities of LOS and Rayleigh channels are well understood, the capacity of the Ricean channel is not straightforward since capacity is not a linear operator. Some exact results are now emerging for finite numbers of antennas [8] but in this Section we will look at the limiting case where $r \rightarrow \infty, t \rightarrow \infty$ and $t / r=\alpha$. Since Rayleigh capacity grows linearly with $m$ and LOS capacity only grows logarithmically, it is intuitively obvious that the normalized Ricean ergodic capacity will approach that of the underlying Rayleigh channel when the number of antennas $(t, r)$ grows large. Also the Ricean ergodic capacity should be greater than that of the underlying Rayleigh channel. Neither of these results appear to be available and so we prove them in this Section.

To begin, note that

$$
\log _{2}\left|I_{r}+\frac{\rho}{t} \boldsymbol{H} \boldsymbol{H}^{*}\right|=\log _{2}\left|I_{r}+\frac{b^{2} \rho}{t} \boldsymbol{H}^{s c}\left(\boldsymbol{H}^{s c}\right)^{*}+\frac{\rho}{t} \boldsymbol{F}\right|,
$$

with $a, b$ defined by (2) and $\boldsymbol{F}$ is the $r \times r$ hermitian matrix,

$$
\boldsymbol{F}=a b\left(\boldsymbol{H}^{s c}\left(\boldsymbol{H}^{s p}\right)^{*}+\boldsymbol{H}^{s p}\left(\boldsymbol{H}^{s c}\right)^{*}\right)+a^{2} \boldsymbol{H}^{s p}\left(\boldsymbol{H}^{s p}\right)^{*} .
$$

From Appendix I, $\boldsymbol{F}$ is a matrix of maximum rank two, with one negative and one positive eigenvalue. The positive eigenvalue, denoted $\lambda_{1}(\boldsymbol{F})$, behaves as below

$$
\lambda_{1}(\boldsymbol{F}) / r t \rightarrow a^{2}
$$

Hence the positive eigenvalue of $\boldsymbol{F}$ grows quadratically with the number of antennas. Despite this, we expect $\boldsymbol{F}$ to have a negligible effect in (15) for large numbers of antennas, since $\boldsymbol{F}$ only has 2 eigenvalues whereas the scattering term has $m$. This is shown below.

\section{A. Lower bound}

We demonstrate in Appendix II that $\forall r, t, \rho, K$,

$$
E\left(\frac{C(K, t, r, \rho)}{\min (t, r)}\right) \geq E\left(\frac{C\left(K=-\infty, t, r, b^{2} \rho\right)}{\min (t, r)}\right) .
$$




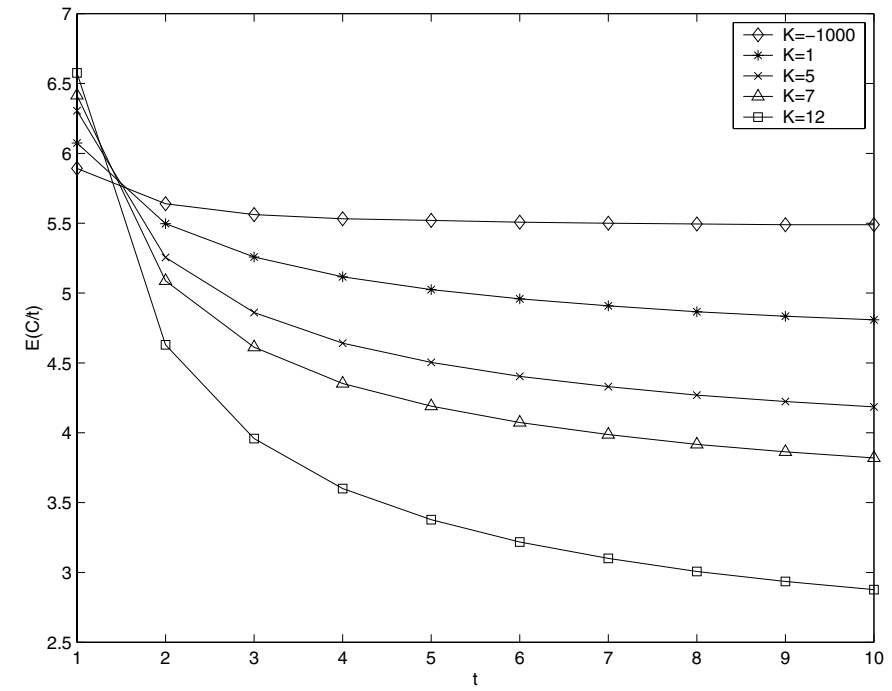

Fig. 1. Ergodic Capacity per antenna with $t / r=1$, Ricean fading and $\mathrm{SNR}=20 \mathrm{~dB}$

\section{B. Upper bound}

We demonstrate in Appendix III that as $t, r \rightarrow \infty$,

$$
E\left(\frac{C(K, t, r, \rho)}{\min (t, r)}\right) \leq E\left(\frac{C\left(K=-\infty, t, r, b^{2} \rho\right)}{\min (t, r)}\right)+\Delta,
$$

where $\Delta \rightarrow 0$ as $t, r \rightarrow \infty$.

Hence, for Ricean channels that are not pure LOS $(K \neq$ $+\infty$ ), the normalized ergodic capacity tends to the normalized ergodic capacity of the scattering component. Hence,

$$
E\left(\frac{C(K, t, r, \rho)}{\min (t, r)}\right) \rightarrow E\left(\frac{C\left(K=-\infty, t, r, b^{2} \rho\right)}{\min (t, r)}\right) .
$$

\section{Simulation results}

Fig. 1 plots the average normalized capacity with $\alpha=1$ for an increasing number of antennas and different $K$-factors. As indicated in our analysis, for $t=1$, the capacity of Ricean channels is higher than the capacity of Rayleigh channels. This trend is inverted for $t, r>1$. For $K=-1000$, the capacity converges rapidly to a limit as $t \rightarrow \infty$, as indicated in [4]. For other values of $K$, the capacity decreases with the number of antennas over this range. As soon as $t>1$, the capacity of the Ricean channel is a decreasing function of $K$.

Fig. 2 shows the behaviour of the normalized capacity of a Ricean channel as the number of antennas grows large. For all values of $K$, the normalized capacity of the Ricean channel tends to the normalized capacity of its scattering component (the lower bound on the capacity). This lower bound is tighter when $K$ is smaller, and for $K=-1000$ it is impossible to discern the simulation from the lower bound.

The upper bound converges slowly to the lower bound and is tight for large values of $K$ and $m$. An explanation for the slowness of convergence can be found in (45) where it is shown that $\Delta$ tends to zero like $\log \left(M+\rho a^{2} r\right) / t$, which itself converges very slowly. Although the upper bound is

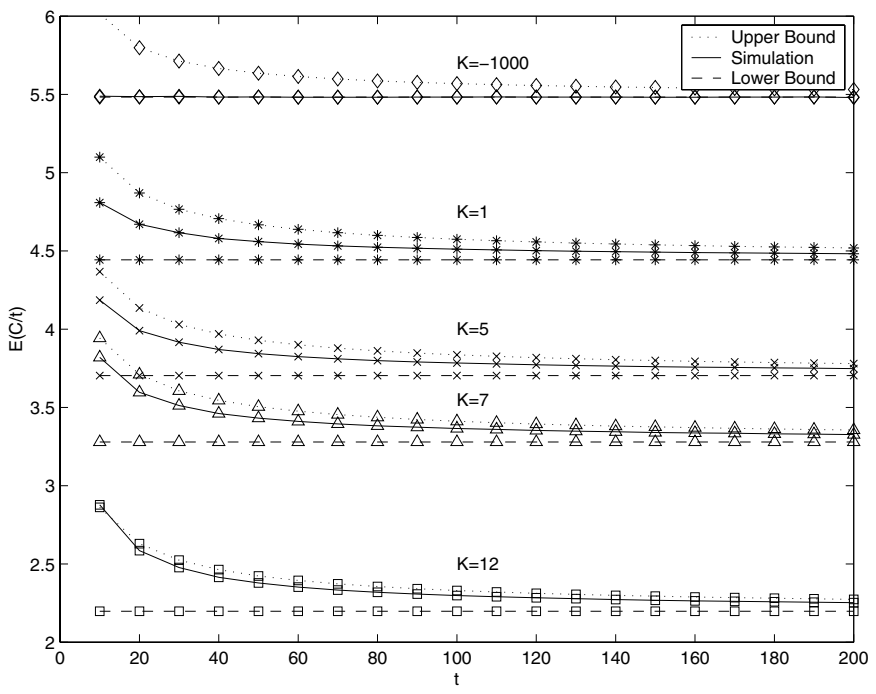

Fig. 2. Ergodic Capacity per antenna with $t / r=1$, Ricean fading and $\mathrm{SNR}=20 \mathrm{~dB}$

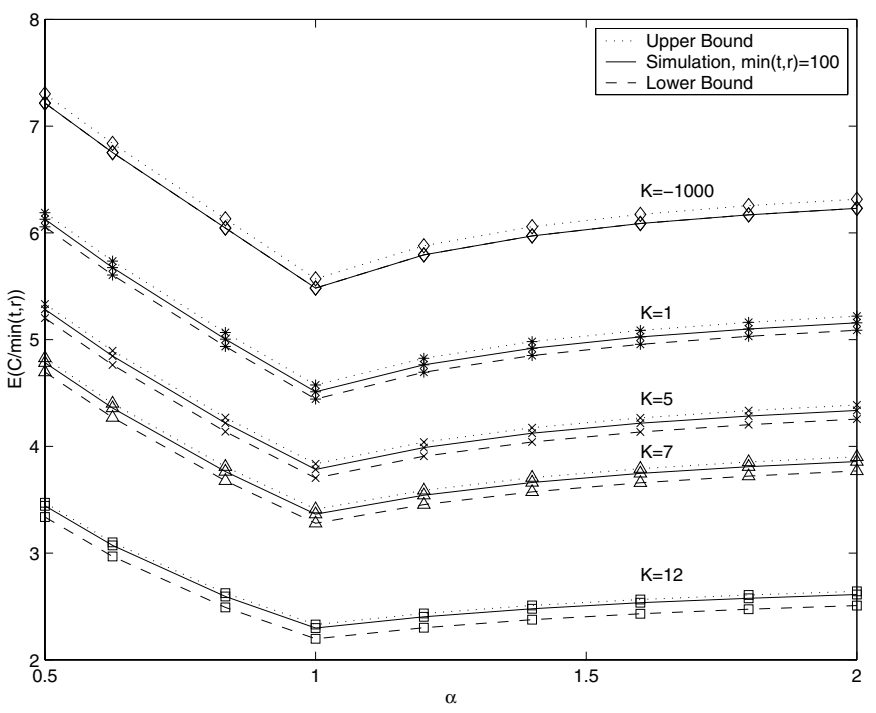

Fig. 3. Ergodic Capacity per antenna as $t, r \rightarrow \infty$ with Ricean fading and $\mathrm{SNR}=20 \mathrm{~dB}$

only strictly valid for a large number of antennas (see the assumptions in (46)), in the simulations it is still correct for values of $t$ as low as 20 , and for $K \leq 12$.

Fig. 3 shows the behaviour of the normalized capacity, for varying $\alpha$, in the asymptotic case of a large number of antennas $(\min (t, r)=100)$. As in Fig. 2, the lower bound is tight for small $K$, whereas the upper bound is tight for large $K$. Note that the tightness of the bounds appears uniform across all $\alpha$ values, indicating that the tightness depends on the ratio $t / r$, and not on their actual values.

The results demonstrate that the upper and lower bounds provide a fast and reliable way to bound the ergodic capacity of a Ricean channel for large $m$, without extensive simulations. Furthermore, depending on the $K$-factor, it is straightforward to deduce which of the bounds is the tightest. 


\section{CONCLUSIONS}

The capacity of the Rayleigh and LOS channels have been studied extensively and are well known, both for a small number of antennas and in the asymptotic case of a large number of antennas. For a Ricean channel, the capacity is more difficult to derive.

For a large number of antennas, the normalized capacity of a Ricean channel tends to the normalized capacity of its Rayleigh component. Precisely, the capacity of the Ricean channel is lower bounded by the capacity of its Rayleigh component and upper bounded by a quantity that tends to the capacity of its Rayleigh component when the number of antennas grows large. The lower bound is valid for any number of antennas, and depending on the choice of a constant $M$, the upper bound can be valid for any number of antennas, or only for a large number of antennas (in which case the upper bound is tighter when the number of antennas grows large). The lower bound is tighter when the $K$-factor is smaller, whereas the upper bound is tighter with increasing $K$. The two bounds allow us to estimate the capacity of a Ricean channel without extensive simulations.

The limiting results for the Ricean channel are useful to observe the speed of convergence towards asymptotic behavior when moderate antenna numbers are employed.

\section{APPENDIX I}

\section{EIGENVALUES OF $\boldsymbol{F}$}

We assume throughout this Appendix that $a \neq 0$.

\section{A. Singular Value Decompositions (SVDs)}

The matrices $\boldsymbol{H}^{s p}\left(\boldsymbol{H}^{s p}\right)^{*}$ and $\boldsymbol{H}^{s c}\left(\boldsymbol{H}^{s p}\right)^{*}$ can be written,

$$
\boldsymbol{H}^{s p}\left(\boldsymbol{H}^{s p}\right)^{*}=t \times(\mathbf{1})_{r, r}
$$

and

$$
\boldsymbol{H}^{s c}\left(\boldsymbol{H}^{s p}\right)^{*}=\left(\sum_{k=1}^{t} h_{i, k}\right)_{i=1 . . r, j=1 . . r} .
$$

Both are rank one matrices and have the following SVDs,

$$
\begin{aligned}
& \boldsymbol{H}^{s p}\left(\boldsymbol{H}^{s p}\right)^{*}=\left(\overrightarrow{v_{1}}\right) r t\left({\overrightarrow{v_{1}}}^{*}\right) \\
& \boldsymbol{H}^{s c}\left(\boldsymbol{H}^{s p}\right)^{*}=\left(\overrightarrow{u_{1}}\right) \sigma\left({\overrightarrow{v_{1}}}^{*}\right) .
\end{aligned}
$$

The singular values are $r t$ and $\sigma$ and the singular vectors are $\overrightarrow{v_{1}}$ and $\overrightarrow{u_{1}}$. These are defined below,

$$
\overrightarrow{v_{1}}=\frac{1}{\sqrt{r}}(\mathbf{1})_{r, 1}
$$

The singular vector $\overrightarrow{u_{1}}$ is given by $\overrightarrow{u_{1}}=\overrightarrow{x_{1}} /\left\|x_{1}\right\|$, where

$$
\begin{gathered}
\overrightarrow{x_{1}}=\left(\sum_{k=1}^{t} h_{1, k}, \sum_{k=1}^{t} h_{2, k}, \ldots, \sum_{k=1}^{t} h_{r, k}\right)^{T} \\
\sigma=\sqrt{r \times \sum_{i=1}^{r}\left\|\sum_{k=1}^{t} h_{i, k}\right\|^{2}},
\end{gathered}
$$

\section{B. Eigenvalues of $\boldsymbol{F}$}

Using the SVDs above, we can write $\boldsymbol{F}$ in (16) as $\boldsymbol{F}=a^{2} r t\left(\overrightarrow{v_{1}}\right)\left({\overrightarrow{v_{1}}}^{*}\right)+a b \sigma\left(\left(\overrightarrow{v_{1}}\right)\left({\overrightarrow{u_{1}}}^{*}\right)+\left(\overrightarrow{u_{1}}\right)\left({\overrightarrow{v_{1}}}^{*}\right)\right)$. Hence $\operatorname{rank}(\boldsymbol{F}) \leq 2$ since $\boldsymbol{F}$ is the sum of two rank 1 matrices, $a^{2} r t\left(\overrightarrow{v_{1}}\right)\left({\overrightarrow{v_{1}}}^{*}\right)+a b \sigma\left(\overrightarrow{v_{1}}\right)\left({\overrightarrow{u_{1}}}^{*}\right)$ and $a b \sigma\left(\overrightarrow{u_{1}}\right)\left({\overrightarrow{v_{1}}}^{*}\right)$. By construction, it follows that any eigenvector, $\vec{k}$, of $\boldsymbol{F}$, associated with the non-zero eigenvalue $\kappa$ satisfies the following,

$$
\left\{\begin{array}{l}
\exists \beta_{1}, \beta_{2} \text { such that } \vec{k}=\beta_{1} \overrightarrow{v_{1}}+\beta_{2} \overrightarrow{u_{1}} \\
\boldsymbol{F} \vec{k}=\kappa \vec{k},
\end{array}\right.
$$

Subsituting for $\boldsymbol{F}$ and $\vec{k}$ and equating coefficients in (28) gives:

$$
\left\{\begin{array}{l}
\beta_{1} a b \sigma\left(\overrightarrow{u_{1}} \vec{v}_{1}\right)+\beta_{2} a b \sigma+\beta_{1} a^{2} r t+\beta_{2} a^{2} r t\left(\overrightarrow{v_{1}} * \overrightarrow{u_{1}}\right)=\kappa \beta_{1} \\
\beta_{1} a b \sigma+\beta_{2} a b \sigma\left(\overrightarrow{v_{1}} * \overrightarrow{u_{1}}\right)=\kappa \beta_{2}
\end{array}\right.
$$

Defining $o={\overrightarrow{v_{1}}}^{*} \overrightarrow{u_{1}}$, and solving (29) for $\kappa$ gives,

$$
\kappa=\frac{\frac{a^{2} r t+a b \sigma\left(o+o^{*}\right)}{2} \pm \ldots}{\frac{\sqrt{\left(a^{2} r t+a b \sigma\left(o+o^{*}\right)\right)^{2}+4(a b \sigma)^{2}\left(1-o o^{*}\right)}}{2} .}
$$

which defines the 2 possibly non-zero eigenvalues of $\boldsymbol{F}$.

\section{Asymptotic eigenvalues of $\boldsymbol{F}$}

Equation (27) indicates that $\sigma \geq 0$ and $E\left(\sigma^{2}\right)=r^{2} \times t$. Furthermore, $\|o\| \leq 1$, so for $r, t \rightarrow \infty,\left(a^{2} r t+a b \sigma(o+\right.$ $\left.\left.o^{*}\right)\right)^{2} \gg 4(a b \sigma)^{2}\left(1-o o^{*}\right)$ and $a^{2} r t \gg\left\|a b \sigma\left(o+o^{*}\right)\right\|$ with probability 1 . Hence, one solution of (30) is positive and the other negative. Since, all other eigenvalues are zero we have the orederd eigenvalues denoted by $\lambda_{r}(\boldsymbol{F})<0=\lambda_{r-1}(\boldsymbol{F})=$ $\ldots=\lambda_{2}(\boldsymbol{F})<\lambda_{1}(\boldsymbol{F})$. Taking the posive square root in (30) gives $\lambda_{1}(\boldsymbol{F}) \sim a^{2} r t+a b \sigma\left(o+o^{*}\right)$ and in the limit

$$
\lambda_{1}(\boldsymbol{F}) / r t \rightarrow a^{2} .
$$

\section{APPENDIX II}

\section{DERIVATION OF THE LOWER BOUND}

Since $\boldsymbol{H} \boldsymbol{H}^{*}$ is a non-central complex Wishart matrix we can use Bartlett's decomposition [9] to give

$$
\boldsymbol{H} \boldsymbol{H}^{*}=b^{2} \boldsymbol{L}^{*} \boldsymbol{L}
$$

where $\boldsymbol{L}$ is upper triangular with diagonal elements denoted $L_{1}, L_{2}, \ldots L_{r}$ which are independent of all other elements. We assume that $r \leq t$ but the proof can easily be adapted to $r>t$ also. The distribution of $L_{1}^{2}$ is non-central chi-squared, $L_{1}^{2} \sim$ $\chi_{2 t}^{2}(\delta)$ with $\delta=\left(a^{2} / b^{2}\right) \operatorname{trace}\left(\boldsymbol{H}^{s p}\left(\boldsymbol{H}^{s p}\right)^{*}\right)$. For $j>1$ the distributions are central chi-squared, $L_{j}^{2} \sim \chi_{2 t-2 j+2}^{2}$. Hence, we have

$$
D=\left|\boldsymbol{I}_{r}+\frac{\rho}{t} \boldsymbol{H} \boldsymbol{H}^{*}\right|=\left|\left[\boldsymbol{I}_{r} \sqrt{\frac{b^{2} \rho}{t}} \boldsymbol{L}^{*}\right]\left[\begin{array}{c}
\boldsymbol{I}_{r} \\
\sqrt{\frac{b^{2} \rho}{t}} \boldsymbol{L}
\end{array}\right]\right|
$$

Using the Cauchy-Binet theorem gives

$$
D=\sum_{\gamma}\left|\boldsymbol{A}_{\gamma} \| \boldsymbol{A}_{\gamma}\right|^{*}=\sum_{\gamma}\left|\boldsymbol{A}_{\gamma}\right|^{2},
$$


where $\boldsymbol{A}_{\gamma}$ is an $r \times r$ submatrix of $\left[\boldsymbol{I}_{r} \sqrt{\frac{b^{2} \rho}{t}} \boldsymbol{L}^{*}\right]$ and $\gamma$ is a subset of $r$ columns from $(1,2, \ldots, 2 r)$.

Now we split the summation into two parts, over $\gamma_{1}$ where the determinants $\left|\boldsymbol{A}_{\gamma_{1}}\right|$ do not involve $L_{1}$ and over $\gamma_{2}$ where the determinants $\left|\boldsymbol{A}_{\gamma_{2}}\right|$ do involve $L_{1}$. Hence

$$
D=\sum_{\gamma_{1}}\left|\boldsymbol{A}_{\gamma_{1}}\right|^{2}+\sum_{\gamma_{2}}\left|\boldsymbol{A}_{\gamma_{2}}\right|^{2}
$$

The only choice of columns which gives determinants involving $L_{1}$ are those where column $r+1$ is selected and column 1 is omitted. Hence the $\boldsymbol{A}_{\gamma_{2}}$ matrices are of the form

$$
\boldsymbol{A}_{\gamma_{2}}=\left[\begin{array}{ccccccc}
0 & \ldots & 0 & \sqrt{\frac{b^{2} \rho}{t}} L_{1} & 0 & \ldots & 0 \\
& & 0 & & & \\
& D_{\gamma_{21}} & \vdots & & D_{\gamma_{22}} & \\
& & 0 & & &
\end{array}\right] .
$$

Hence $\left|\boldsymbol{A}_{\gamma_{2}}\right|^{2}=\frac{b^{2} \rho}{t} L_{1}^{2}\left|D_{\gamma_{2}}\right|^{2}$ where $D_{\gamma_{2}}=\left[D_{\gamma_{21}} D_{\gamma_{22}}\right]$ and $D=\sum_{\gamma_{1}}\left|\boldsymbol{A}_{\gamma_{1}}\right|^{2}+\frac{b^{2} \rho}{t} L_{1}^{2} \sum_{\gamma_{2}}\left|D_{\gamma_{2}}\right|^{2}=X+L_{1}^{2} Y$. Exactly the same analysis holds for the Rayleigh case, except $L_{1}^{2} \sim \chi_{2 t}^{2}$.

To summarize,

$$
\begin{gathered}
D^{\text {Ricean }}=X+\chi_{2 t}^{2}(\delta) Y \\
D^{\text {Rayleigh }}=X+\chi_{2 t}^{2} Y
\end{gathered}
$$

where $X, Y$ are positive random variables with $X, Y$ independent of the $\chi^{2}$ variables. Hence

$$
\begin{aligned}
& E(C(\boldsymbol{H}))=E\left(\log _{2}(X)\right)+E\left(\log _{2}\left(1+\chi_{2 t}^{2}(\delta) Y / X\right)\right) \\
& E\left(C\left(b \boldsymbol{H}^{s c}\right)\right)=E\left(\log _{2}(X)\right)+E\left(\log _{2}\left(1+\chi_{2 t}^{2} Y / X\right)\right) .
\end{aligned}
$$

Now $\chi_{2 t}^{2}(\delta)$ is stochastically greater than $\chi_{2 t}^{2}$. Hence $E\left(f\left(\chi_{2 t}^{2}(\delta)\right)\right) \geq E\left(f\left(\chi_{2 t}^{2}\right)\right)$ for any increasing function $f$ and $E(C(\boldsymbol{H})) \geq E\left(C\left(b \boldsymbol{H}^{s c}\right)\right)$ as required.

\section{APPENDIX III}

\section{DERIVATION OF THE UPPER BOUND}

Defining

$$
\boldsymbol{A}=\boldsymbol{I}_{r}+\frac{b^{2} \rho}{t} \boldsymbol{H}^{s c}\left(\boldsymbol{H}^{s c}\right)^{*},
$$

we have the normalized capacity as

$$
\frac{C}{t}=\frac{1}{t} \log _{2}(|\boldsymbol{A}+\tilde{\boldsymbol{F}}|)=\frac{1}{t} \log _{2}\left(\prod_{i=1}^{r} \lambda_{i}(\boldsymbol{A}+\tilde{\boldsymbol{F}})\right),
$$

where $\tilde{\boldsymbol{F}}=\frac{\rho}{t} \boldsymbol{F}$ and $\lambda_{i}(\boldsymbol{A}+\tilde{\boldsymbol{F}})$ are the eigenvalues of the hermitian positive definite matrix $\boldsymbol{A}+\tilde{\boldsymbol{F}}$, ordered so that $0 \leq$ $\lambda_{r}(\boldsymbol{A}+\tilde{\boldsymbol{F}}) \leq \ldots \leq \lambda_{1}(\boldsymbol{A}+\tilde{\boldsymbol{F}})$. Combining Weyl's theorem [10] and results from Appendix I leads to

$$
\begin{aligned}
\lambda_{r}(\boldsymbol{A}+\tilde{\boldsymbol{F}}) \leq & \lambda_{r-1}(\boldsymbol{A})+\lambda_{2}(\tilde{\boldsymbol{F}})=\lambda_{r-1}(\boldsymbol{A}) \\
\lambda_{r-1}(\boldsymbol{A}+\tilde{\boldsymbol{F}}) \leq & \lambda_{r-2}(\boldsymbol{A})+\lambda_{2}(\tilde{\boldsymbol{F}})=\lambda_{r-2}(\boldsymbol{A}) \\
& \vdots \\
\lambda_{2}(\boldsymbol{A}+\tilde{\boldsymbol{F}}) \leq & \lambda_{1}(\boldsymbol{A})+\lambda_{2}(\tilde{\boldsymbol{F}})=\lambda_{1}(\boldsymbol{A}) \\
\lambda_{1}(\boldsymbol{A}+\tilde{\boldsymbol{F}}) \leq & \lambda_{1}(\boldsymbol{A})+\lambda_{1}(\tilde{\boldsymbol{F}})
\end{aligned}
$$

Therefore,

$$
\begin{aligned}
\frac{C}{t} & \leq \frac{1}{t} \log _{2}\left(\lambda_{r-1}(\boldsymbol{A}) \ldots \lambda_{1}(\boldsymbol{A})\left(\lambda_{1}(\boldsymbol{A})+\lambda_{1}(\tilde{\boldsymbol{F}})\right)\right. \\
& =\frac{1}{t} \log _{2}\left(\prod_{i=1}^{r} \lambda_{i}(\boldsymbol{A})\right)+\frac{1}{t} \log _{2}\left(\frac{\lambda_{1}(\boldsymbol{A})+\lambda_{1}(\tilde{\boldsymbol{F}})}{\lambda_{r}(\boldsymbol{A})}\right)
\end{aligned}
$$

Now write

$$
\Delta=\frac{1}{t} \log _{2}\left(\frac{\lambda_{1}(\boldsymbol{A})+\lambda_{1}(\tilde{\boldsymbol{F}})}{\lambda_{r}(\boldsymbol{A})}\right),
$$

and, since $\lambda_{j}(\boldsymbol{A}) \geq 1$ for any $\mathrm{j}$,

$$
\Delta \leq \frac{1}{t} \log _{2}\left(\lambda_{1}(\boldsymbol{A})+\frac{\rho}{t} \lambda_{1}(\boldsymbol{F})\right) .
$$

It is known that the eigenvalues of $\boldsymbol{A}$ are bounded as $r, t \rightarrow \infty$ [11]. Therefore, $\exists M$ such that $\lambda_{1}(\boldsymbol{A}) \leq M$ and when $t \rightarrow \infty$,

$$
\Delta \leq \frac{1}{t} \log _{2}\left(M+\rho r\left(\lambda_{1}(\boldsymbol{F}) /(r t)\right)\right) \rightarrow 0,
$$

since $\lambda_{1}(\boldsymbol{F}) /(r t) \rightarrow a^{2}$. This concludes the demonstration. From [11] we know that

$$
\lambda_{1}(\boldsymbol{A}) \rightarrow 1+b^{2} \rho(1+\sqrt{\min (t, r) / \max (t, r)})^{2},
$$

as $t, r \rightarrow \infty$ with $t / r=\alpha$. This provides the smallest value for $M$ that can be used and gives the bound that we use in the simulations.

\section{REFERENCES}

[1] G.J. Foschini, M.J. Gans, "On limits of wireless communication in a fading environment when using multiple antennas," Wireless Personal Communications, vol. 6, no. 3, pp. 311-335, March 1998.

[2] I.E. Telatar, "Capacity of multi-antenna gaussian channels," European Transactions on Telecommunications, vol. 10, no. 6, pp. 585-595, 1999.

[3] P.J. Smith and M. Shafi, "On a Gaussian approximation to the capacity of wireless MIMO systems," Proc. IEEE International Conference on Communications (ICC2002), pp. 406-410, New York, April 28-May 2, 2002.

[4] E. Biglieri and Giorgio Tarico, "Large-System Analysis of MultipleAntenna System capacities," Journal of Commiunications and Networks., vol. 5, No. 2, pp. 96-103, June. 2003.

[5] P.F. Driessen and G. J. Foschini, "On the capacity formula for multipleinput-multiple-output wireless channels: a geometric approach," IEEE Trans. Commun., vol. 47, No. 2, pp. 173-176, Feb. 1999.

[6] F. R. Farrokhi, G. J. Foschini, A. Lozano and R.A. Valenzuela, "Linkoptimal space-time processing with multiple transmit and receive antennas," IEEE Communications Letters, vol. 5, no. 3, pp. 85-87, March 2001.

[7] S.K. Jayaweera and H.V. Poor, "On the capacity of multi-antenna systems in the presence of Ricean fading," Proc. IEEE Vehicular Technology Conference (VTC2002), pp. 1963-1967, Vancouver, September 24-28, 2002.

[8] P.J. Smith and L.M. Garth, "Exact capacity distribution for Dual MIMO systems in Ricean fading," in press IEEE Communications Letters.

[9] R.J. Muirhead, Aspects of Multivariate Statistical Theory, New York, John Wiley \& Sons Inc., 1982.

[10] R.A. Horn and C.R. Johnson, Matrix Theory, Cambridge, Cambridge University Press, 1985.

[11] A. Edelman, Eigenvalues and Condition Numbers of Random Matrices, PhD thesis, MIT, 1989. 